\title{
High-Power Single-Mode Antiresonant Reflecting Optical Waveguide-Type Vertical-Cavity Surface-Emitting Lasers
}

\author{
Delai Zhou, Member, IEEE, and Luke J. Mawst, Senior Member, IEEE
}

\begin{abstract}
Antiresonant reflecting optical waveguide (ARROW) techniques are employed in vertical cavity surface emitting lasers (VCSELs) to achieve high-power single-mode emission. Using the effective-index method and fiber mode approximation, the cold-cavity lateral modal behavior for the circular shaped ARROW VCSEL demonstrates significant reduction of radiation loss from that of a single antiguide, while maintaining strong discrimination against high-order modes. The circular-waveguide is created by selective chemical etching and two-step metal-organic chemical vapor deposition growth, with proton implantation used to confine the current injection to the low-index core region. A single-mode $\mathrm{CW}$ power of $7.1 \mathrm{~mW}$ has been achieved from an 8 $\mu \mathrm{m}$ diameter ARROW device (index step $\Delta n=0.05$, emission at $\lambda_{0}=980 \mathrm{~nm}$ ) with a far-field FWHM of $10^{\circ}$. Larger aperture $(12 \mu \mathrm{m})$ devices exhibit multimode operation at lower drive currents with a maximum single-mode continuous-wave output power of $4.3 \mathrm{~mW}$.
\end{abstract}

Index Terms-Diode lasers, epitaxial growth, quantum-well lasers, semiconductor growth, semiconductor lasers, vertical-cavity surface-emitting lasers.

\section{INTRODUCTION}

$\mathbf{T}$ HE ABILITY to achieve high-power single-mode vertical-cavity surface-emitting lasers (VCSELs) is of great interest for many applications such as telecommunications, optical storage, and laser printing, etc. While the conventional small-aperture (diameter $<4 \mu \mathrm{m}$ ) VCSEL's maximum output power (single-mode) is limited to about $5 \mathrm{~mW}$ [1], there are increasing interests in investigating larger aperture, lower resistance, lower current-density devices for higher continuous-wave $(\mathrm{CW})$ output powers. Due to the fact that a larger waveguide generally supports multiple modes, the focus is to effectively increase the modal discrimination in favor of the fundamental mode. Recently, several approaches [2]-[5] exhibited about 6-mW single-mode output from large-aperture $(>8 \mu \mathrm{m})$ positive-index guided (oxide-confined) VCSELs. On the other hand, negative-index waveguides (antiguides) with a large built-in modal discrimination (higher order modes have

Manuscript received May 10, 2002.

D. Zhou was with the Reed Center for Photonics, Department of Electrical Computer Engineering, University of Wisconsin-Madison, Madison, WI 53706-1691 USA. He is now with Phosistor Technology Inc., Pleasanton, CA 94588 USA.

L. J. Mawst is with was with the Reed Center for Photonics, Department of Electrical Computer Engineering, University of Wisconsin-Madison, Madison, WI 53706-1691 USA (e-mail: mawst@engr.wisc.edu).

Digital Object Identifier 10.1109/JQE.2002.805107 significantly higher edge radiation losses) is a natural choice for large spot-size single-mode operation. Although antiguided VCSELs have been demonstrated to be capable of operating single mode up to very high driving currents [6]-[9], output powers were low, due to the high-edge radiation losses for the fundamental mode.

In order to reduce the radiation loss in one-dimensional (1-D) antiguided waveguide systems, a few pairs of lateral reflectors has been introduced to reflect back the lateral leaky waves from the antiguided core [10], [11]. When these reflectors meet the antiresonant condition, similar to the VCSEL's longitudinal distributed Bragg reflector (DBR) case, the antiguide's radiation loss will be minimized. These types of structures are generally called antiresonant reflecting optical waveguide (ARROW) and have been demonstrated successfully in edge-emitting lasers with $0.3-\mathrm{W}$ CW single-mode operation [12]. In addition, a large index-step $(\Delta n>0.025)$ design provides sufficient mode stability against carrier- and thermally-induced index variations [13]. Effectively, the ARROW reflectors can be viewed as a truncated photonic lattice and the wider antiguided-core as a $d e$ fect of the lattice. The supported modes can be classified into two types: 1) highly localized modes in the vicinity of the defect with a very low loss and 2) nonlocalized modes traveling through the lattice with high loss [14], [15]. Thus, traditional ARROW laser structures can be considered a defect laser with one pair of reflectors, and gain placed in the low- index core region to select the low-loss localized leaky mode. More simply, we could surround the antiguided core with only one high-index antiresonant layer (i.e., half-pair DBR). This type of device is referred to as the simplified antiresonant reflecting optical waveguide, or S-ARROW.

Previously, we have demonstrated single-mode operation from large aperture $(12 \mu \mathrm{m}$ diameter $)$ traditional ARROW and S-ARROW VCSELs [16], [17], although output powers were relatively low (1-2 mW CW). Here, we present a design analysis based on cold-cavity simulation using the effective-index approximation. Device results for 8-12- $\mu \mathrm{m}$ diameter circular-shaped ARROW VCSELs with ring-reflectors, demonstrate CW single-mode output powers as high as $7.1 \mathrm{~mW}$.

\section{Device Design and Modal Behavior}

For a single antiguide with a core index of $n_{0}$ and index step of $\Delta n=n_{1}-n_{0}$, the $m$ th-order leaky mode possess a radiating 


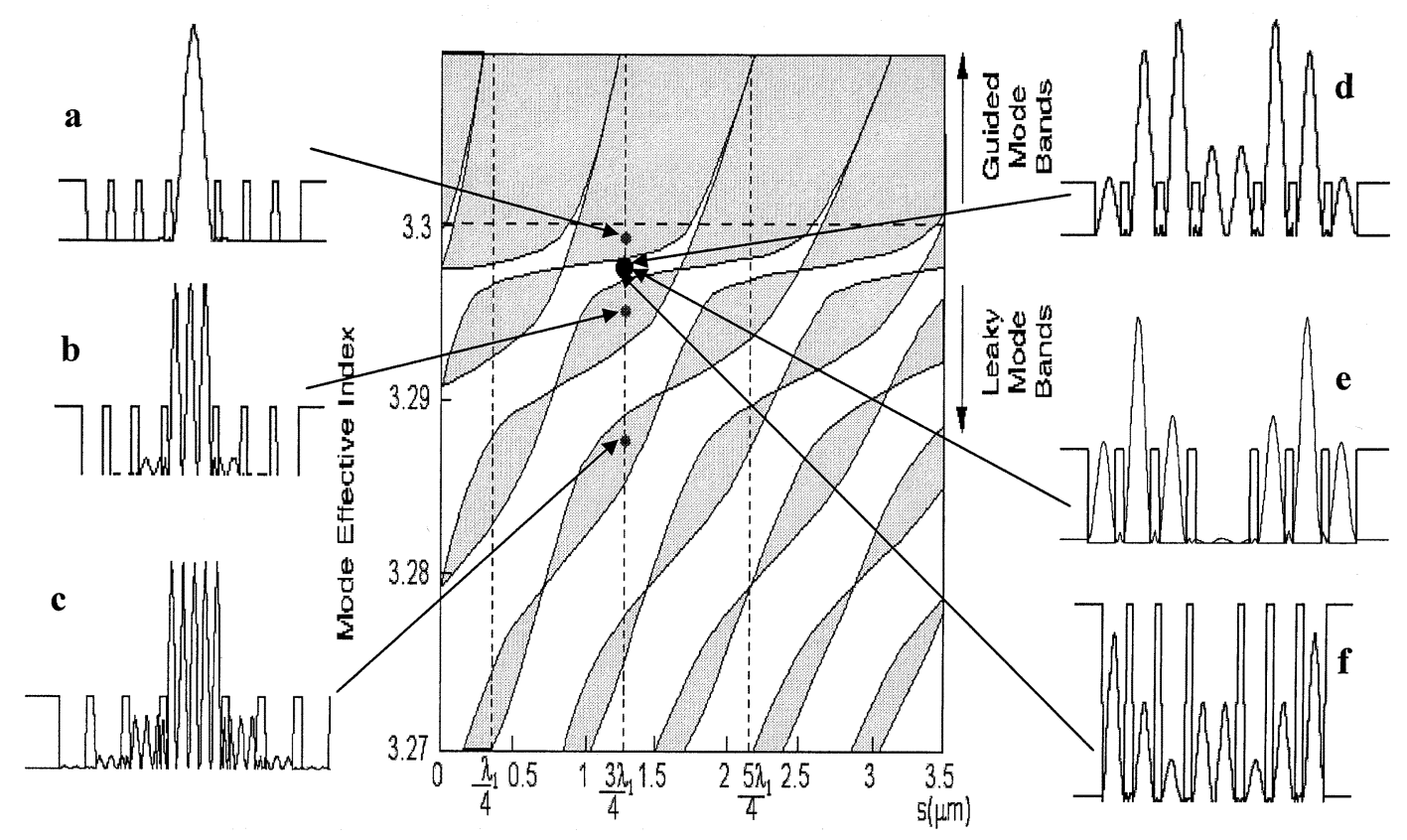

Fig. 1. Calculated allowed solution bands for a 1-D (infinite) photonic lattice as a function of high-index region width, $s$. The modal effective index locations for various modes (a-f) of a finite (13-layer) defect incorporated lattice, is also shown. Modes a, b, c correspond to localized states formed within the forbidden gaps of the infinite lattice. Modes d-f correspond to propagating solutions within the allowed solution bands of the infinite lattice (low index $n_{0}=3.3$, high index $\left.n_{1}=3.35\right)$.

field in the high-index $\left(n=n_{1}\right)$ cladding regions characterized by a lateral wavelength $\lambda_{1}$ that can be approximated by

$$
\lambda_{1}=\frac{\lambda_{0}}{\left[n_{1}^{2}-n_{0}^{2}+\frac{(m+1)^{2} \lambda_{0}^{2}}{4 d^{2}}\right]^{1 / 2}}
$$

and a lateral radiation loss $\alpha$

$$
\alpha=\frac{(m+1)^{2} \lambda_{0}}{\left(n_{1}^{2}-n_{0}^{2}+\frac{\lambda_{0}^{2}}{4 d^{2}}\right)^{1 / 2}}
$$

where $\lambda_{0}$ is the vacuum wavelength and $d$ is the low-index "core" width [18], [19]. From above equations, it's clear that: 1) different modes have different lateral wavelengths and 2) higher order modes have much higher radiation losses than the fundamental mode. In order to reduce this lateral radiation loss, we need to confine the propagation of the lateral traveling wave that has a wavelength of $2 d$ in the low-index region (if $d>2 \mu \mathrm{m}$ and $\Delta n>0.02)$ [18] and $\lambda_{1}$ in the high-index region $(n=$ $\left.n_{1}\right)$. Specifically, if each layer is designed to be antiresonant (quarter-wave thickness) to the incident wave, such as forming a lateral DBR, the reflection is a maximum and the loss is a minimum. Considering the fundamental mode, we can surround the antiguide core with two layers that have indexes of $n_{1}$ and $n_{0}$ and width of $p * \lambda_{1} / 4$ and $q * d / 2$, respectively, where $p$ and $q$ are odd integer numbers in order to minimize its loss. Other modes with different lateral wavelengths will not meet the antiresonant conditions in either one or both of the reflecting layers, and therefore, their losses are not minimized.

An alternative way to view the ARROW concept is that of a defect within a photonic lattice, which acts to confine the photon propagation and therefore reduces the lateral radiation loss. For an infinite 1-D periodic dielectric structure, Bloch function analysis can be used to determine the propagating Bloch-wave solutions [20]. Bands of propagating solutions are separated by forbidden gaps, corresponding to evanescently decaying solutions. In active photonic lattice devices, great interest is focused on how to control the modal behavior, e.g., single-lateral-mode emission is usually desired. For this purpose, we calculate the modal effective index (defined as $n_{\text {eff }}=\beta / k_{0}$, where $k_{0}$ is the vacuum wave vector and $\beta$ is the propagation constant normal to the lattice plane) of Bloch-wave solutions for the infinite lattice as a function of the lattice geometry, such as varying the high-index region width, $s$, as shown in Fig. 1. The group of allowed bands in which the modal effective index lies between $n_{0}$ and $n_{1}$ are referred to as the guided-mode bands. The leaky-mode bands originate from the modes confined to low-index regions (antiguides) of the lattice and have a modal effective index lower than $n_{0}$, also shown in the $\beta$-s band diagram of Fig. 1. The modes of the finite array can be shown to lie within the allowed mode bands of the infinite lattice. In an antiguided array structure, gain is selectively placed in the low-index lattice sites of the array, allowing selection of modes within the leaky-mode bands. Note that this is very different from conventional photonic lattice structures, consisting of dielectric material with air holes, where gain cannot be placed in the low-index regions of the lattice.

The modes supported within the allowed leaky-mode bands of a finite array suffer significant edge radiation losses. Increasing the lattice element number is one effective way to reduce this loss. Another way is to utilize the fact that a defect state, which lies inside the forbidden gap, is not allowed to propagate through lattice, resulting in very low loss for the finite lattice situation. One established way to produce the forbidden gap mode is to introduce a defect into the photonic 


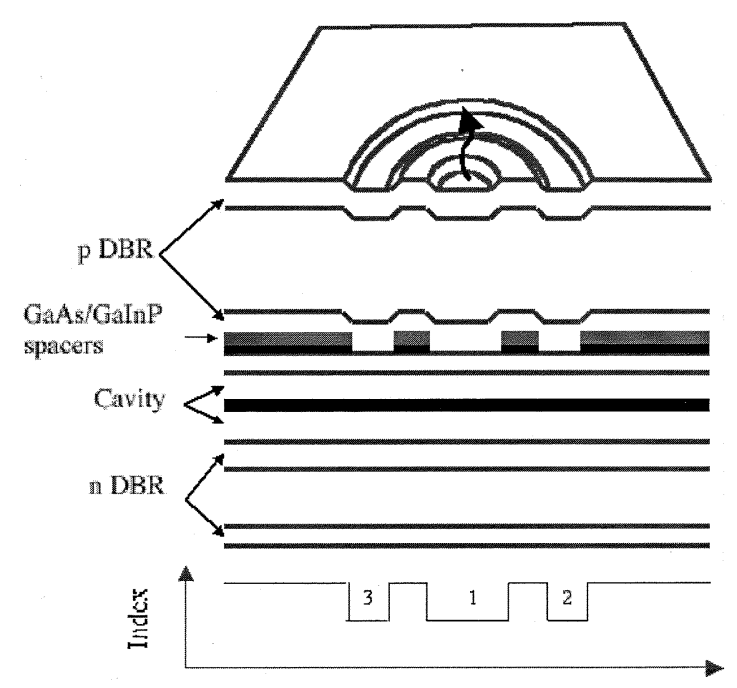

Fig. 2. 3-D cross-sectional view of a circular shaped ARROW VCSEL with a 1-pair lateral reflector. The high- and low-index ring reflectors are defined by chemically etching a thin GaAs-GaInP spacer layer, followed by regrowth. The resulting lateral index-step is also shown, where regions 1,2 , and 3 correspond to areas where the spacer layers are removed.

lattice, just as the donor/acceptor cases in the electronic bandstructure. For a 1-D lattice, if one of the low-index lattice sites is changed, e.g., becomes twice as wide, a localized leaky mode will be formed inside this larger defect and exhibit relatively low radiation loss.

Photonic-lattice-based microcavity laser structures localize light to a defect mode, generally within a forbidden gap lying between the guided-mode bands. This type of localized mode can exhibit a high cavity $\mathrm{Q}$ and a small modal volume, making the structure ideal for the waveguide of a microcavity laser. Villeneuve et al. and Krauss et al. [21]-[23] demonstrated the possibility of forming defect states in the forbidden gaps between leaky-mode bands. Such photon states are less localized than the guided-mode defect states and, due to radiation losses, exhibit a lower cavity Q. Although the transmission characteristics of photonic lattices with a leaky-mode defect were analyzed, active devices have not been investigated.

For simplicity, we consider here a 13-layer 1-D lattice, with the center low-index lattice-site width doubled (defect). The resulting modal profiles (selected modes) are plotted in Fig. 1, and the locations on the band diagram are also labeled. Among them, modes a, b, c, are expected to have the lowest losses because their modal effective index lies inside the gap. Other modes, such as modes d, e, f, have a modal effective index lying in the allowed bands; therefore, the losses are relatively high. It is evident that (see Fig. 4) the ARROW-type device can be viewed as such a defect-incorporated active (truncated) photonic lattice, allowing selection of the leaky fundamental mode because of its low radiation loss. Proper design of the ARROW laser structure, as discussed below, allows only the lowest-order defect mode (mode a of Fig. 1) to reach laser threshold, since higher order gap modes and modes within the allowed bands suffer significantly higher edge radiation losses.

Here, we only consider the circular-shaped device with one pair of ring reflectors for ARROW VCSEL, of which the cross-sectional view is shown in Fig. 2. Other than the layers

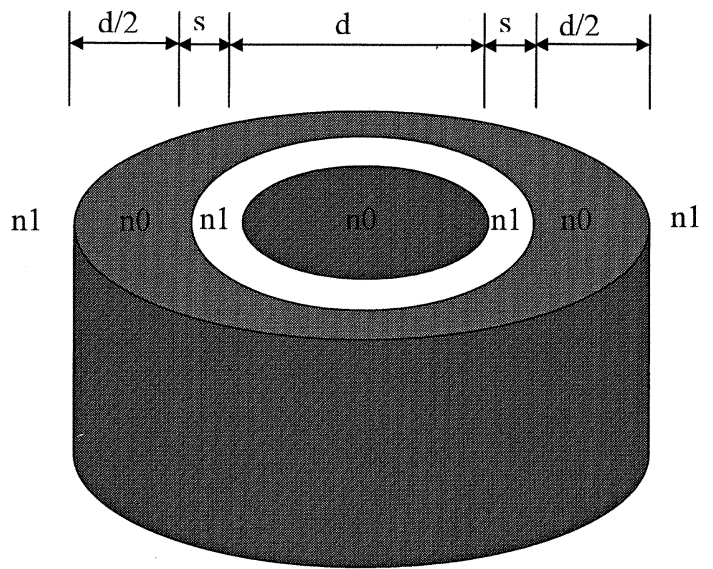

Fig. 3. Effective index model and fiber-mode approximation of circular-shaped ARROW VCSEL: core diameter is $d$; high-index reflector width is $s$; index step $\Delta n=n_{1}-n_{0}$.

labeled as "spacer layers" that are needed to form the lateral core and reflectors, the structure is the same as a conventional 980-nm-emitting VCSEL with n-DBR of AlAs-GaAs, cavity of InGaAs-GaAs-AlGaAs and p-DBR of AlAs-AlGaAs.

Shifting the cavity resonant wavelength $\lambda_{\text {res }}$ of a VCSEL is equivalent to a change of its effective-index $n_{\text {eff }}$ with the approximate relation [24]

$$
\frac{\Delta n_{\mathrm{eff}}}{n_{\mathrm{eff}}}=\frac{\Delta \lambda_{\mathrm{res}}}{\lambda_{\mathrm{res}}} .
$$

Therefore, by selectively removing the spacer layers in the desired low-index regions of Fig. 2 (region 1, 2, and 3) to blue-shift the resonance wavelength compared to their neighboring regions with the spacer layers, circular-shaped ARROW VCSELs are created. In our structure, the lateral index step $\Delta n$ is designed to be in the range of $0.025-0.1$, corresponding to a spacer layer thickness of about 12-45 $\mathrm{nm}$ [assuming an average index of 3.3 in (3)], which is a sufficiently large value to insure mode stability. For this work, the width of the outer low-index reflector is fixed to be one-quarter wavelength, namely $d / 2$. In order to study the modal behavior of such 2-D ARROW-type VCSELs and find the antiresonance position, we vary the width of the high-index reflectors. Experimentally, we have targeted its width to be $\sim 5 / 4 \lambda_{1}$ for ease of fabrication, which in turn can be deduced from the 1-D equation in (1), provided that the effective index step is known. We expect the above approximation of using the 1-D design rule for a 2-D structure to be quantitatively correct since the radial field profile of the supported modes of a 2-D antiguided large aperture cylindrical waveguide have the form of first- and second-kind Bessel functions, which can be approximated as cosine and sine waves.

We use a 2-D effective-index model and fiber-mode approximation to simulate the 3-D ARROW-type VCSEL structures [16], [17]. In this approximation, the antiguided core and reflector regions are simply represented by the effective indices $n_{0}$ and $n_{1}$ respectively, calculated from the 1-D transverse layered structure in each region. The 3-D structure is then transferred into a 2-D "ARROW fiber," shown in Fig. 3. In the following calculations, the parameters are chosen as: $d=8 \mu \mathrm{m}, n_{0}=3.3$, 

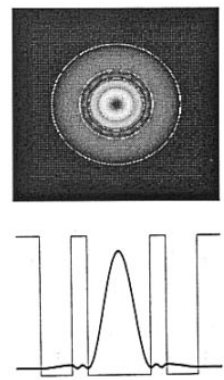

(a)
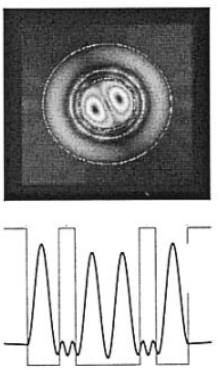

(d)
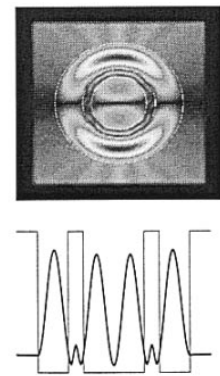

(b)
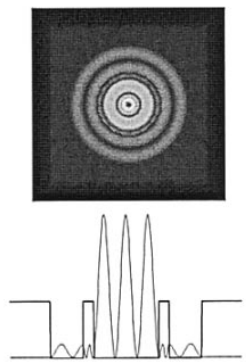

(e)
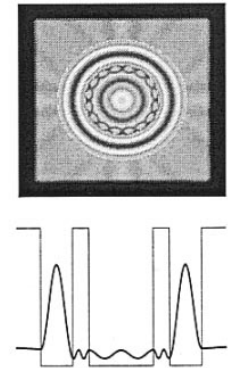

(c)

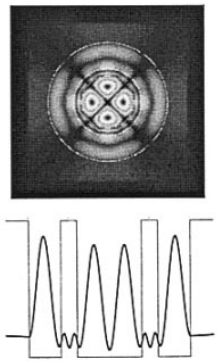

(f)
Fig. 4. Computed 2-D transverse mode profiles of the ARROW VCSEL with the associated 1-D scan underneath. (a)-(e) Correspond to modes 4, 5, 6, 7, and 8, respectively. (f) $L P_{21}$ mode.

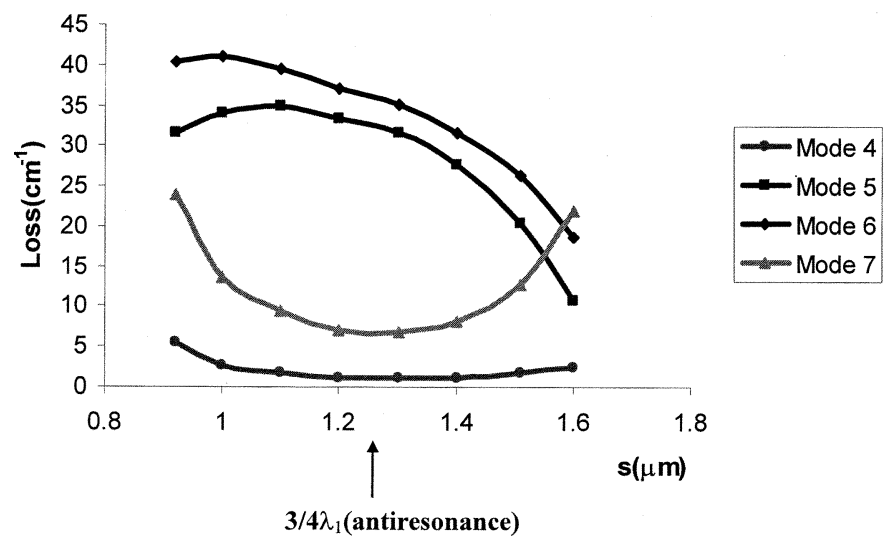

Fig. 5. Computed modal radiation losses for the ARROW VCSEL with an 8 $\mu \mathrm{m}$-diameter core and an index step of $\Delta n=0.05$. The lowest loss modes from Fig. 4, are included as a function of high-index reflector spacing.

$n_{1}=3.35,(\Delta n=0.05)$, and $\lambda_{0}=0.98 \mu \mathrm{m}$. From (1), the lateral wavelength in the high-index region is $\lambda_{1}=1.7 \mu \mathrm{m}$.

The computed 2-D scalar modes, shown in Fig. 4, include the fundamental mode (localized, mode 4) and several low-loss higher order modes (traveling, modes 5, 6, 7). Other higher order localized modes are found to have relatively large loss and not to be discussed here. The mode number is defined by counting the standing wave nulls of its 1-D counterpart, as shown in Fig. 4. In Fig. 5, the corresponding modal radiation losses are calculated as the function of the high-index reflector width $s$, while the low-index reflector is set at $4 \mu \mathrm{m}(d / 2)$. As expected, the fundamental mode's radiation loss is significantly reduced (minimum value $\sim 1 \mathrm{~cm}^{-1}$ ) compared with that of a single antiguide $\left(\sim 22 \mathrm{~cm}^{-1}\right)$ with the same diameter $(8 \mu \mathrm{m})$ and index step

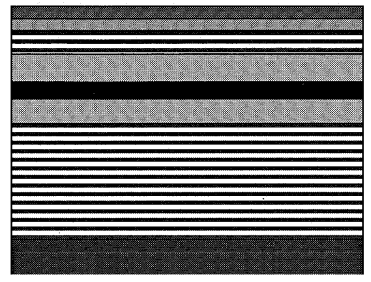

(a)

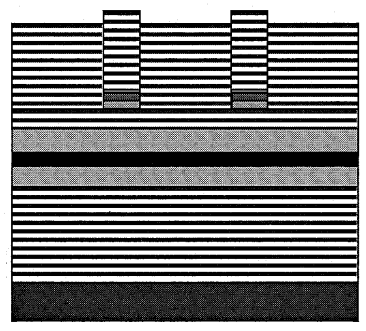

(c)

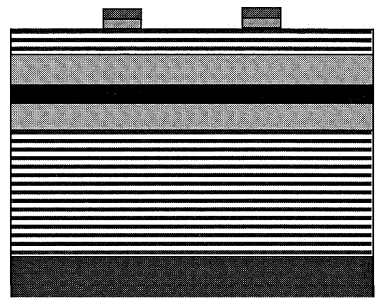

(b)

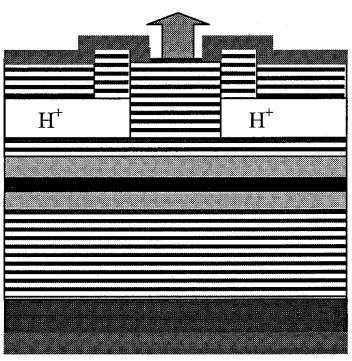

(d)
Fig. 6. Fabrication process flow diagram of the ARROW VCSEL. (a) First MOCVD growth of the n-DBR, cavity, one pair p-DBR and index layers. (b) Selective wet chemical etch to form the ARROW VCSEL reflector and core regions. (c) MOCVD regrowth of the remaining part of the p-DBR. (d) p-side metallization and liftoff, $\mathrm{H}^{+}$ion implantation and $\mathrm{n}$ side metallization.
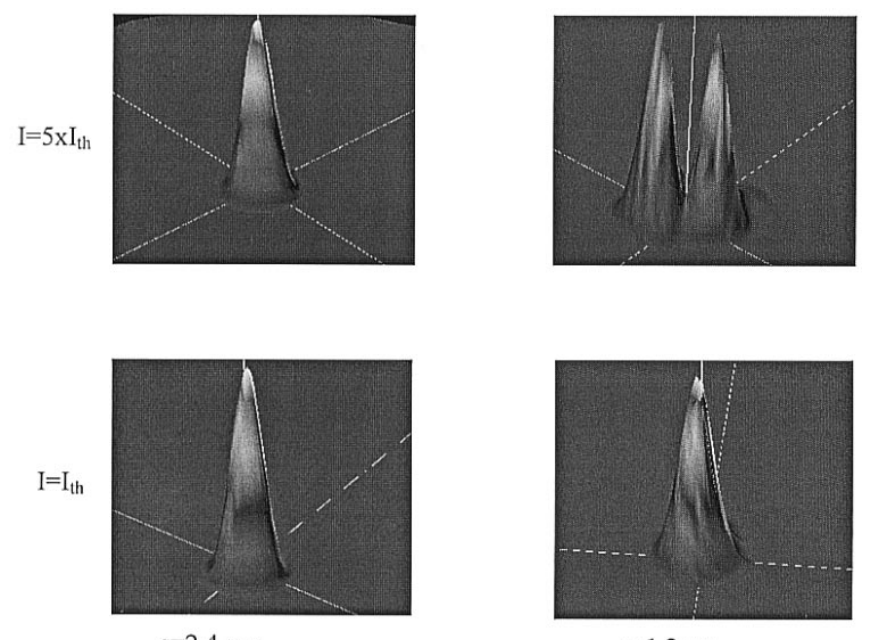

$\mathrm{s}=1.2 \mu \mathrm{m}$

Fig. 7. Measured 3-D views of the far-field patterns for the ARROW VCSELs measured under CW conditions near $I_{t h}$ and $5 \times I_{t h}$. Two devices are shown, with high-index reflector widths, $s=1.2$ and $s=2.4 \mu \mathrm{m}$.

$(\Delta n=0.05)$. Although higher order modes also experience reduced losses, the effect is not as strong as that of the fundamental mode. In fact, the ratio of the losses between the next lowest loss, higher order mode (mode 7) and the fundamental mode is increased to $>5$, while in the single-antiguide, the ratio is $\sim 2.5$. From our analysis, the antiresonant position for the fundamental mode corresponds to its lowest loss region, which occurs at $s \sim 1.3 \mu \mathrm{m}\left(\sim 3 / 4 \lambda_{1}\right)$ in Fig. 5, indicating good agreement with the 1-D calculation, using (1). In addition, a relatively large region exists around the antiresonance where strong high-order mode discrimination occurs, with low loss for the fundamental 

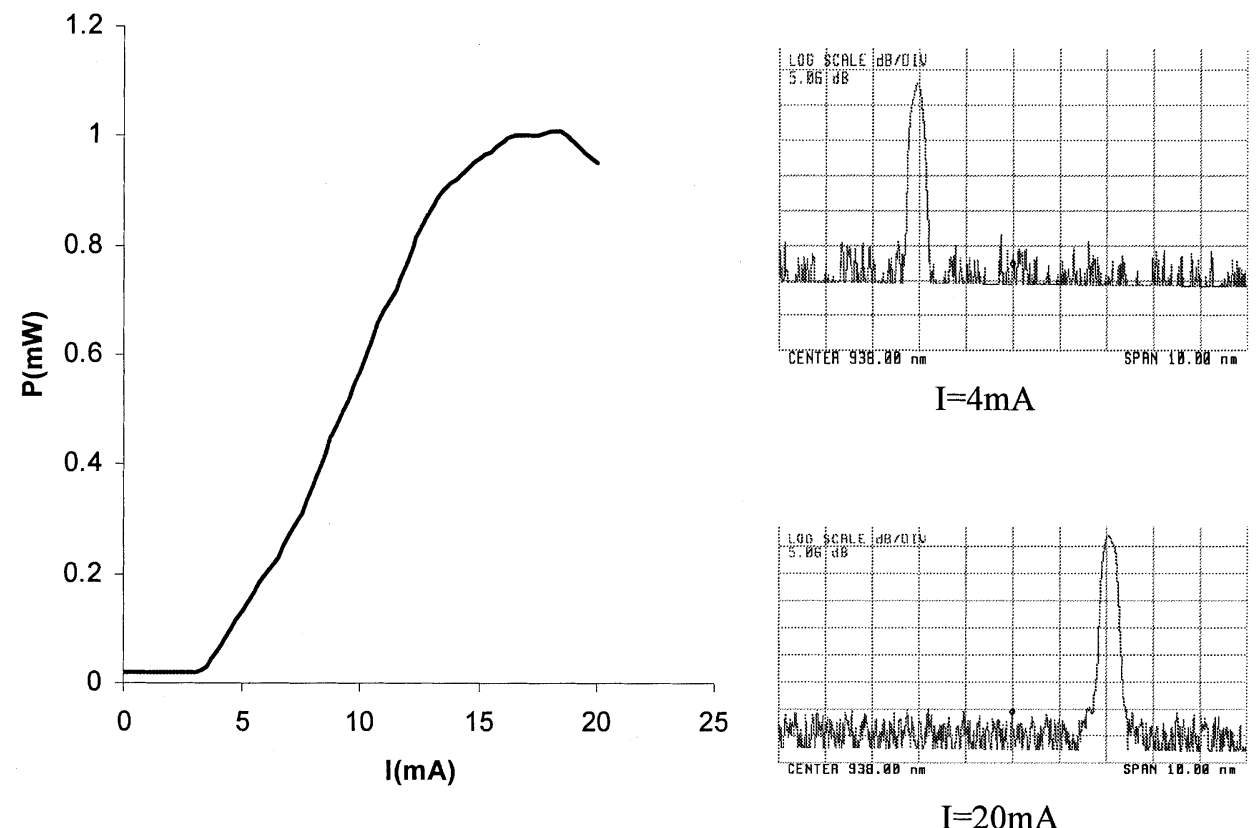

Fig. 8. CW P-I curve and spectra of an unoptimized ARROW VCSEL with core width, $d=12 \mu \mathrm{m}$, and $\Delta n=0.05$ and high-index reflector width, $s=2.4 \mu \mathrm{m}$. The device remains single-mode up to thermal rollover at 1-mW output power. The center of the spectral scan is $938 \mathrm{~nm}$ and the span is $10 \mathrm{~nm}$, with a vertical scale of $5 \mathrm{~dB} /$ division.

mode. Away from antiresonance, improved modal discrimination is achieved at the expense of higher fundamental mode loss. Similar behavior occurs for different index-step structures (i.e., $\Delta n=0.1$ or 0.025 ), although the losses decrease with the increase of the index step. While polarization is not included in this simplified model, previous full-wave FDTD studies of the modal behavior of the S-ARROW VCSEL indicates that polarization-dependent losses can occur [25].

While the strong mode dependent losses provide the primary mechanism to discriminate against higher order modes, other factors such as gain spatial hole burning effects and carrier- and thermally-induced index variations need to be considered above laser threshold. While such a comprehensive analysis has not been performed for the 3-D ARROW-type VCSEL structure, previous studies [13] on 1-D ARROW structures have indicated that tight current confinement to the low-index core region is desirable to maintain fundamental mode stability to drive currents high above laser threshold. Gain redistribution and index variations above threshold, which leads to focusing or defocusing of high-order modes, promotes multimode behavior.

\section{DEVICE FABRICATION AND EXPERIMENTAL RESULTS}

The ARROW VCSEL fabrication sequence is shown schematically in Fig. 6. After the first MOCVD growth including n-type DBR, one wave cavity, two pairs of p-type DBRs, and index layers $\operatorname{GaInP}(12 \mathrm{~nm})$ and $\operatorname{GaAs}(20 \mathrm{~nm})$, we use photolithography to pattern the wafer and wet chemical etch ( $\mathrm{HCl}: \mathrm{H}_{2} \mathrm{O}$ for the GaInP layer and $\mathrm{H}_{3} \mathrm{PO}_{4}: \mathrm{H}_{2} \mathrm{O}_{2}: \mathrm{H}_{2} \mathrm{O}$ for the GaAs layer) to selectively remove the thin spacer layers, forming the index step and circular ARROW structure. The second growth involves the regrowth of the remaining top p-type DBR and $p^{+}$GaAs contact layer. Following the p-metal
(Ti:Pt:Au) liftoff to open the output window in the top contact region, $\mathrm{H}^{+}$proton implantation is used to confine the current into the central low-index core region.

Initially, we designed and fabricated large aperture ARROW VCSEL devices [17] with a core diameter $=12 \mu \mathrm{m}$ and $\Delta n=0.05$. The modal behavior was studied by varying the reflector width, $s$, although they were not optimized devices. For some device geometries, such as $s=1.2 \mu \mathrm{m}$, the devices were found to operate multimode as the current increases well beyond the threshold. Other devices with different reflector width, such as $s=2.4 \mu \mathrm{m}$, however, remained single mode through the whole operating range until thermal rollover. The measured 3-D CW far-field, CW power-current $(P-I)$ curve, and spectrum are shown in Figs. 7 and 8, respectively. They confirm a CW 1-mW single fundamental mode emission at 936-940 nm, with a side-lobe suppression ratio of over $30 \mathrm{~dB}$. Because the lateral wave $\lambda_{1} \sim 1.7 \mu \mathrm{m}, 1.2 \mu \mathrm{m}$ corresponds to approximately $3 / 4 \lambda_{1}$, while $2.4 \mu \mathrm{m}$ is close to $3 / 2 \lambda_{1}$. However, uncertainty in the actual built-in index step makes it difficult to determine the lateral wavelength, and hence the antiresonant point can shift relative to that calculated in Fig. 5. The threshold currents (3-5 mA) for devices with different values of $s$ are very similar, since the loss for the fundamental mode loss does not have much variation (one reason is that the outer low-index reflector is always antiresonant to the fundamental mode). In addition, there are some other factors that may affect the device thresholds and output power, such as the unoptimized number of DBR pairs (23) and cap layer thickness (3/4 wave). In addition, the misalignment (in the wrong direction) between the optical mode and gain peak wavelength is believed to be a major limitation. Therefore, as the device operates under CW operation, the efficiency and maximum output powers are low.

After we optimized our VCSEL design, by adjusting the spectral gain peak to be shorter than the cavity resonance, improved 


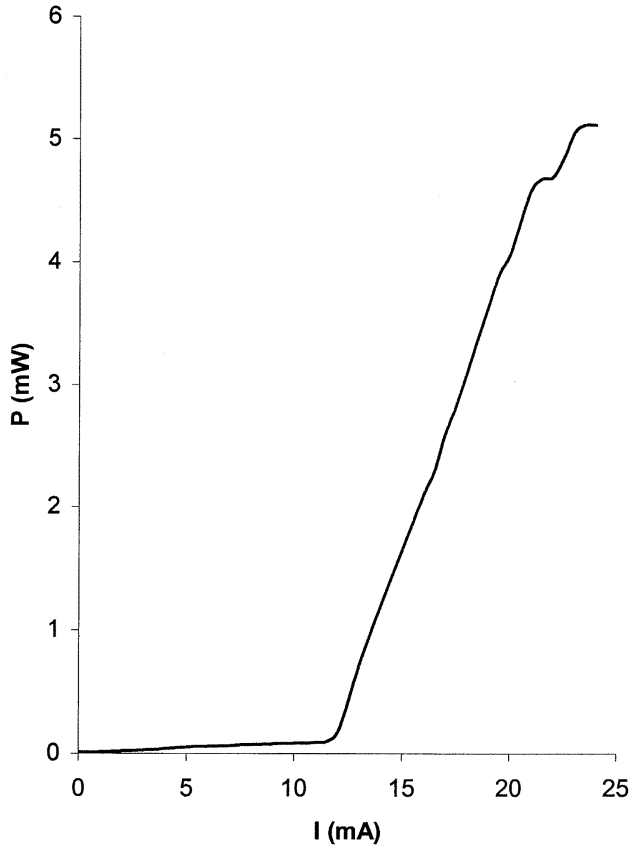

Fig. 9. Measured CW $P-I$ curve of an optimized $12-\mu \mathrm{m}$-diametercore ARROW VCSEL with $\Delta n=0.05$ and high-index reflector width, $s=2.4 \mu \mathrm{m}$. Single-mode output power is measured up to approximately 4 $\mathrm{mW}$.

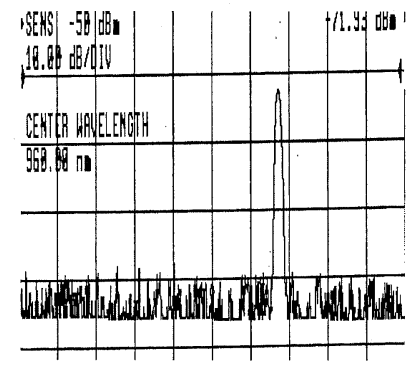

$\mathrm{I}=19 \mathrm{~mA}$

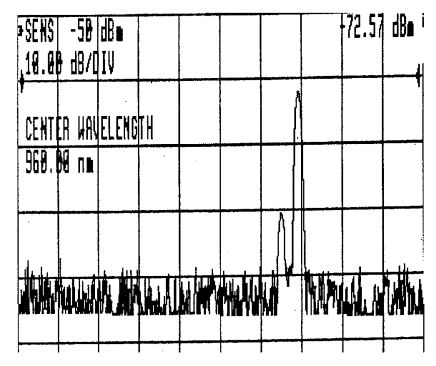

$\mathrm{I}=20 \mathrm{~mA}$
Fig. 10. Measured CW spectra characteristics of the $12 \mu \mathrm{m}$ diameter ARROW VCSEL, as described for Fig. 9, indicating CW single-mode output up to $4 \mathrm{~mW}$. The center of the spectral scan is $960 \mathrm{~nm}$, with a span of $20 \mathrm{~nm}$ and a vertical scale of $10 \mathrm{~dB} /$ division.

performance is achieved. Devices were fabricated with two different core sizes of 12- and 8- $\mu \mathrm{m}$ diameters and with high-index reflector width of $s=2.4 \mu \mathrm{m}$. ARROW VCSELs with $12-\mu \mathrm{m}$ -diameter core width begin to lase at a threshold current of 10-12 mA, but then turn multimode at about $2 \times$ threshold, with a maximum single mode output power in the range of $4-5 \mathrm{~mW}$. Even for such a device with a large injection area (20- $\mu \mathrm{m}$ diameter) defined by the implantation, the device still emits $\sim 4 \mathrm{~mW}$ $\mathrm{CW}$ single fundamental-mode power, as shown in Fig. $9(P-I$ curve) and Fig. 10 (spectrum).

For 8- $\mu$ m-diameter core ARROW VCSELs, the CW spectrum in Fig. 11 shows single-mode emission up to $23 \mathrm{~mA}$, with over 20-dB side mode suppression ratio, corresponding to 7.1-mW CW single-mode output power, as indicated in the $P-I$ curve of Fig. 12. Furthermore, under pulsed operation (200-ns pulses at $50 \mathrm{kHz}$ ), single-mode emission is observed up to
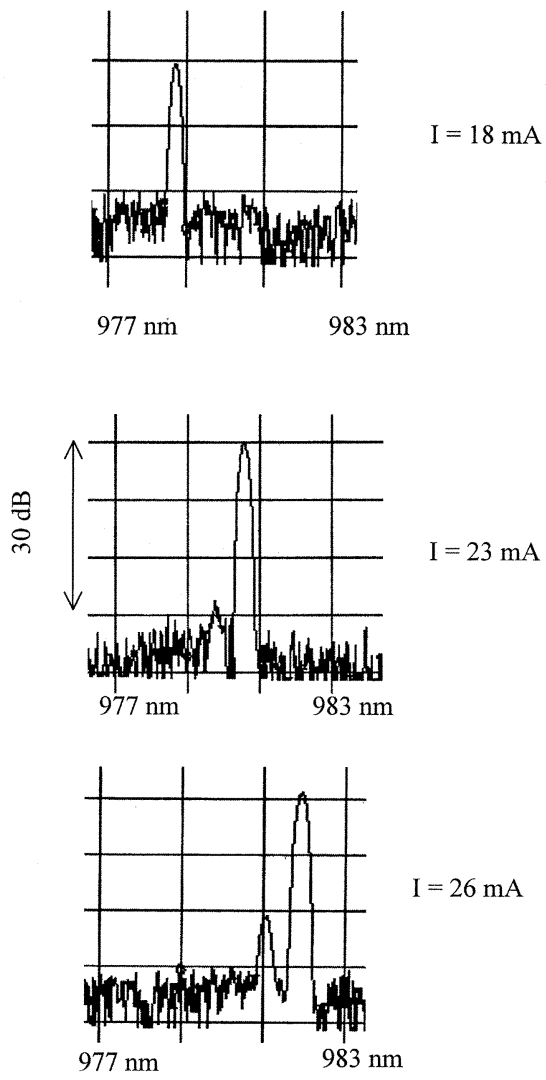

Fig. 11. Measured spectrum for an $8-\mu$ m-diameter ARROW VCSEL with $\Delta n=0.05$ under $\mathrm{CW}$ operation. Single-mode operation is observed up to approximately 7-mW CW (with $S M S R=20 \mathrm{~dB}$ ).

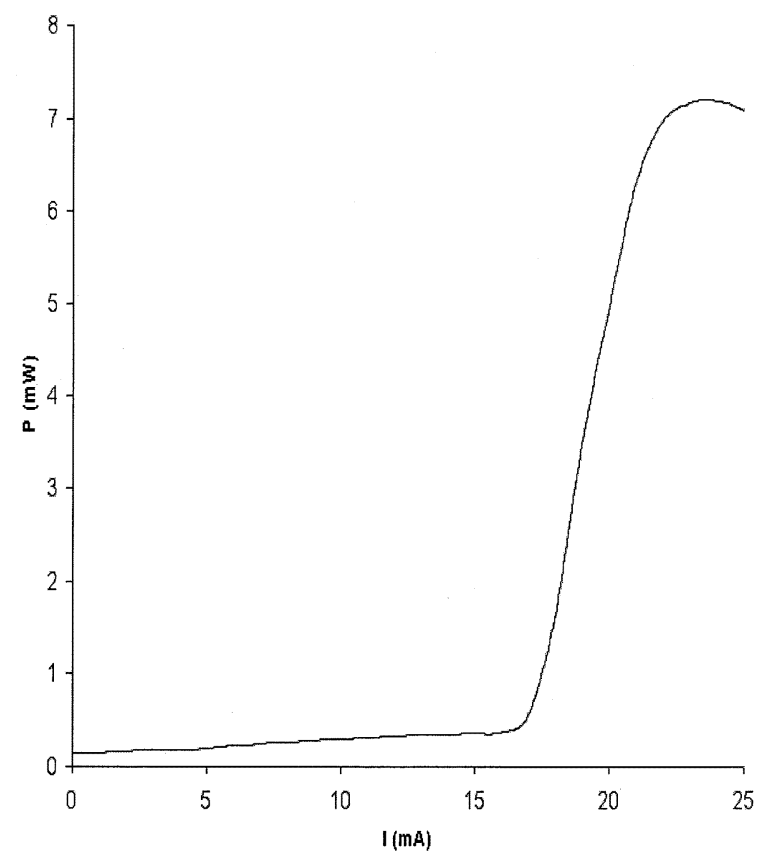

Fig. 12. Measured CW P-I curve for the 8- $\mu$ m-diameter ARROW VCSEL, as described in Fig. 11.

significantly higher drive currents ( $>37 \mathrm{~mA})$, indicating that the thermal effects play an important role in the multimode onset, as expected. Presumably, the next lowest loss, higher order mode (i.e., mode 7 from Fig. 5) reaches threshold at 


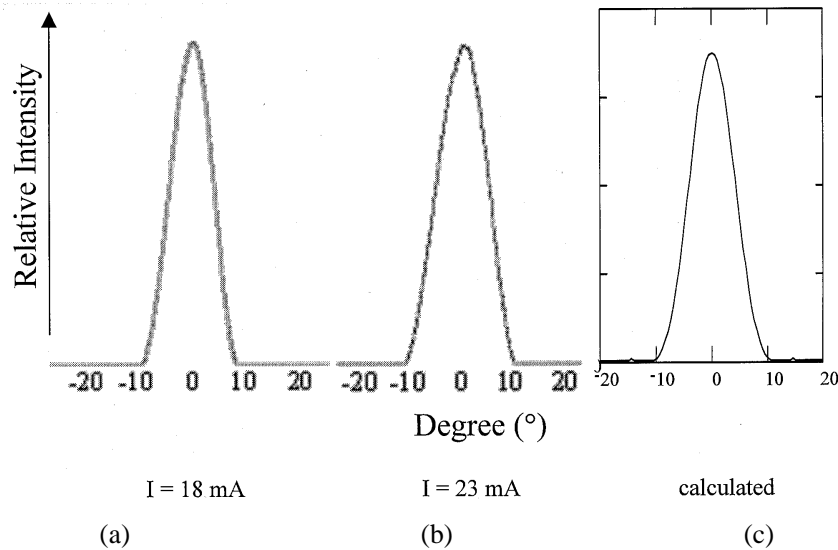

Fig. 13. 1-D slice of the far-field radiation pattern for the 8 - $\mu$ m-diameter ARROW VCSEL with $\Delta n=0.05$. (a), (b) Measured under CW operation. (c) Calculated.

high drive currents, due to thermal self-focussing and gain-spatial-hole-burning. However, a detailed above-threshold analysis is required to study the mode competition at high drive currents above the threshold of the fundamental mode (i.e., mode 4 from Figs. 4 and 5). The CW far-field profile, shown in Fig. 13, indicates that the ARROW VCSEL is lasing in a single-fundamental mode (mode 4 ) with a FWHM angle of about $10^{\circ}$, which agrees well with the calculated value. Further improvements can be obtained, such as varying the device index step and effectively removing excessive heating utilizing a junction-down geometry. Although the apparent threshold current is relatively large, at $16 \mathrm{~mA}$, we believe that this is not the true threshold, but a "snap-on" phenomenon, after comparing threshold values to previous fabrication runs (discussed above). This artificial "threshold" can be caused by some unintentional (saturable) absorbing defects during the tight processing procedures, such as misalignment between the device and the implantation mask. Once the defect absorption gets saturated, the VCSEL starts to "lase" and results in a "snap-on" effect of the $P-I$ curve, with an apparent high threshold and efficiency. Optimization of the proton-implantation processing procedure is required to eliminate this undesirable effect.

\section{CONCLUSIONS}

In conclusion, we have demonstrated the ARROW VCSELs for high-power single-mode operation. Theoretical studies indicate that the low-loss leaky fundamental mode could be selected over other high-order, higher radiation loss, modes over a broad range of device parameters for ARROW VCSEL structures. The simple 1-D calculation can be used to determine the ARROW-type VCSEL's antiresonance and agrees well with a 2-D effective index model and fiber mode approximation. A maximum CW single-mode power of $7.1 \mathrm{~mW}$ at a wavelength of $980 \mathrm{~nm}$ was obtained from an $8-\mu \mathrm{m}$-diameter device with a built-in index of $\Delta n=0.05$. By optimizing the device design such as aperture diameter, reflector geometry and dimension, built-in lateral index step, and DBR parameters, higher single-mode power over $10 \mathrm{~mW}$ is expected to be achieved in the future.

\section{REFERENCES}

[1] C. Jung, R. Jager, M. Grabherr, P. Schnitzer, R. Michalzik, B. Weigl, S. Muller, and K. J. Ebeling, " $4.8 \mathrm{~mW}$ single mode oxide confined topsurface emitting vertical-cavity laser diodes," Electron. Lett. , vol. 33, p. 1790, 1997.

[2] H. J. Unold, M. Kicherer, S. W. Z. Mahmoud, R. Jager, R. Michalzik, and K. J. Ebeling, "Spatially varied anti-resonant DBR design for large-area single-mode VCSEL's," Proc. 2000 IEEE 17th Int. Semiconductor Laser Conf., pp. 57-58, Sept. 2000.

[3] K. D. Choquette, A. J. Fischer, K. M. Geib, G. R. Hadley, A. A. Allerman, and J. J. Hindi, "High single mode operation from hybrid ion implanted/selectively oxidized VCSEL's," Proc. 2000 IEEE 17th Int. Semiconductor Laser Conf., pp. 63-64, Sept. 2000.

[4] S. W. Z. Mahmoud, H. J. Unold, W. Schmid, R. Jager, R. Michalzik, and K. J. Ebeling, "Analysis of longitudinal mode wave guiding in verticalcavity surface-emitting lasers with long monolithic cavity," Appl. Phys. Lett., vol. 78, pp. 586-588, 2001.

[5] A. J. Fischer, K. D. Choquette, W. W. Chow, A. A. Allerman, D. D. Serkland, and K. M. Geib, "High single mode power observed from a coupled-resonator vertical-cavity laser diode," Appl. Phys. Lett., vol. 79, pp. 4079-4081, 2001

[6] Y. A. Wu, G. S. Li, R. F. Nabiev, K. D. Choquette, C. Caneau, and C. J. Chang-Haisnain, "Single-mode, passive antiguide vertical cavity surface emitting laser," IEEE J. Select. Topics Quantum Electron., vol. 1, p. 629, 1995.

[7] T. H. Oh, M. R. McDaniel, D. L. Huffaker, and D. G. Deppe, "Cavity-induced antiguiding in a selectively oxidized vertical-cavity surface-emitting laser," IEEE Photon. Technol. Lett., vol. 10, pp. 12-14, Jan. 1998.

[8] K. D. Choquette, G. R. Hadley, H. Q. Hou, K. M. Geib, and B. E. Hammons, "Leaky mode vertical cavity lasers using cavity resonance modification," Electron. Lett., vol. 34, p. 991, 1998.

[9] B. S. Yoo, H. Y. Chu, M. S. Park, and E. H. Lee, "Stable transverse mode emission in vertical-cavity surface-emitting lasers antiguided by amorphous GaAs layer," Electron. Lett., vol. 32, pp. 116-117, 1996.

[10] L. J. Mawst, D. Botez, C. Zmudzinski, and C. Tu, "Antiresonant reflecting optical waveguide-type, single-mode diode lasers," Appl. Phys. Lett., vol. 61, pp. 503-505, 1992.

[11] T. L. Koch, U. Koren, G. D. Boyd, P. J. Corvini, and M. A. Duguay, "Antiresonant reflecting optical waveguide for III-V integrated optics," Electron. Lett., vol. 23, pp. 244-245, 1987.

[12] L. J. Mawst, D. Botez, C. Zmudzinski, and C. Tu, " $0.3 \mathrm{~W}$ CW single-spatial-mode operation from large-core ARROW-type diode lasers," Electron. Lett., vol. 28, pp. 1793-1795, 1992.

[13] L. J. Mawst, D. Botez, R. F. Nabiev, and C. Zmudzinski, "Above-threshold behavior of high-power, single-mode antiresonant reflecting optical waveguide diode lasers," Appl. Phys. Lett., vol. 66, p. 7, 1995.

[14] D. Zhou, A. Napartovich, N. N. Elkin, D. V. Vysotsky, and L. J. Mawst, "Modal characteristics of 2-D leaky-wave coupled vertical-cavity surface-emitting laser arrays," presented at the SPIE Photonics West, Optoelectronics 2002, Vertical Cavity Surface Emitting Lasers VI, paper 4649-26.

[15] C. A. Zmudzinski, D. Botez, and L. J. Mawst, "Simple description of laterally resonant, distributed-feedback-like modes of arrays of antiguides," Appl. Phys. Lett., vol. 60, pp. 1049-1051, 1992.

[16] D. Zhou and L. J. Mawst, "Simplified-antiresonant reflecting optical waveguide-type vertical-cavity surface-emitting lasers," Appl. Phys. Lett., vol. 76, p. 1659, 2000.

[17] - "Antiresonant reflecting optical waveguide (ARROW) type VCSEL," Proc. IEEE LEOS 2000, vol. 2, Nov. 2000.

[18] R. W. Engelmann and D. Kerps, "Leaky modes in active three-layer slab waveguides," Proc. Inst. Elect. Eng., pt. I, vol. 127, pp. 330-336, Dec. 1980.

[19] D. Botez, L. J. Mawst, and G. Peterson, "Resonant leaky-wave coupling in linear arrays of antiguides," Electron. Lett., vol. 24, pp. 1328-1330, 1988.

[20] P. Yeh, Optical Waves in Layered Media. New York: Wiley, 1988, ch. 6.

[21] P. R. Villeneuve, S. Fan, S. G. Johnson, and J. D. Joannopoulos, "Three-dimensional photon confinement in photonic crystals of low-dimensional periodicity," Proc. Inst. Elect. Eng.-Optoelectron., vol. 145, p. 384, 1998.

[22] H. Benisty, C. Weisbuch, D. Labilloy, M. Rattier, C. J. M. Smith, T. F. Krauss, R. M. De La Rue, R. Houdre, U. Oesterle, C. Jouanin, and D. Cassagne, "Optical and confinement properties of two-dimensional photonic crystals," J. Lightwave Technol., vol. 17, p. 2063, 1999. 
[23] M. Rattier, H. Benisty, C. J. M. Smith, A. Beraud, D. Cassagne, C. Jouanin, T. F. Krauss, and C. Weisbuch, "Performance of waveguide-based two-dimensional photonic-crystal mirrors studied with Fabry-Perot resonators," IEEE J. Quantum Electron., vol. 37, p. 237, 2001.

[24] G. R. Hadley, "Effective index model for vertical-cavity surface-emitting lasers," Opt. Lett., vol. 20, pp. 1483-1485, 1995.

[25] T.-W. Lee, S. Hagness, D. Zhou, and L. J. Mawst, "Modal characteristics of ARROW-type vertical-cavity surface-emitting lasers," IEEE Photon. Technol. Lett., vol. 13, pp. 770-772, 2001.

Delai Zhou (M'01) was born in Shenyang, China, in 1975. He received the B. S. degree in nuclear physics from Peking University, Peking, China, in 1995, and the M.S.E.E. and Ph.D. degrees in 1998 and 2002. respectively, from the Department of Electrical and Computer Engineering, University of WisconsinMadison. His dissertation research involved the development of high-power single-mode VCSELs and two-dimensional phase-locked VCSEL arrays utilizing the antiguided structures.

In 2002, he joined Phosistor Technology Inc., Pleasanton, CA, as a member of Technical Staff.
Luke J. Mawst (M'88-SM'93) was born in Chicago, IL, in 1959. He received the B.S. degree in engineering physics and the M.S. and Ph.D. degrees in electrical engineering from the University of Illinois at Urbana-Champaign in 1982, 1984, and 1987, respectively. His dissertation research involved the development of index-guided semiconductor lasers and laser arrays grown by MOCVD.

In 1987, he joined TRW Inc., Redondo Beach, CA, where he was a Senior Scientist in the Research Center, engaged in design and development of semiconductor lasers using MOCVD crystal growth. The primary focus of this work has been to develop high-output-power coherent laser-diode sources. He coinvented the resonate optical waveguide (ROW) antiguided array and has contributed to its development as a practical source of high coherent power. He also developed a novel single-laser structure (the ARROW laser) as a source for coupling high powers into fibers. He has also been involved in the development of two-dimensional coherent surface-emitting arrays, vertical-cavity surface emitters, and distributed-feedback laser structures. He is currently an Associate Professor at the University of Wisconsin-Madison, where he is involved in the development of novel semiconductor laser structures using the A1-free InGaAs-GaAs-based material system. he has authored or coauthored over 100 technical papers and holds eight patents.

Dr. Mawst is an IEEE Lasers and Electro-Optics Society (LEOS) Distinguished Lcturer. He was the recipient of the TRW Group Level Chairman's Award. 